VOL. 65 (2002) [503-510]

\title{
FORMULAE ASSOCIATED WITH 5, 7, 9 AND 11 SQUARES
}

\section{Pierre Barrucand and Michael D. Hirschhorn}

Let $r_{k}(n)$ denote the number of representations of $n$ as the sum of $k$ squares. We give elementary proofs of relations between $r_{k}(n)$ and $r_{k}(m)$ where $n=4^{\lambda} m$ and $4 \nmid m$, when $k=5,7,9$ and 11 . These relations, which were first stated without proof by Stieltjes, are of the form $r_{k}(n)=C r_{k}(m)$ where $C$ depends on $\lambda$ and on the residue of $m$ modulo 8 . They have recently been included by $S$. Cooper in a more complete description of the relations between $r_{k}(n)$ and $r_{k}\left(n^{\prime}\right)$ where $n^{\prime}$ is the squarefree part of $n$, when $k=5,7,9$ and 11 .

\section{INTRODUCTION}

Let $r_{k}(n)$ denote the number of representations of $n$ as the sum of $k$ squares. Due to the work of Ramanujan and many others, $r_{k}(n)$ is well understood for $k$ even, but is less well so for $k$ odd. In two recent papers $([1,2])$, Cooper has given the relationship between $r_{k}(n)$ and $r_{k}\left(n^{\prime}\right)$ where $n^{\prime}$ is the square-free part of $n$ for $k=5$ and $k=7$ and for $k=9$ when $n^{\prime} \equiv 5(\bmod 8)$ and for $k=11$ when $n^{\prime} \equiv 7(\bmod 8)$. In so doing, he needs the relations between $r_{k}(n)$ and $r_{k}(m)$ where $n=4^{\lambda} m$ and $4 \nmid m$. The object of this note is to give elementary proofs of these relations, which were apparently first stated, without proof, by Stieltjes in the 1880 's. They are as follows.

If $n=4^{\lambda} m$ where $4 \nmid m$ then

$$
\begin{gathered}
r_{5}(n)=C r_{5}(m) \quad \text { where } C= \begin{cases}\left(8^{\lambda+1}-1\right) / 7 & \text { if } m \equiv 1(\bmod 8), \\
\left(5 \times 8^{\lambda+1}+9\right) / 49 & \text { if } m \equiv 5(\bmod 8), \\
\left(4 \times 8^{\lambda}+3\right) / 7 & \text { if } m \equiv 2 \text { or } 3(\bmod 4),\end{cases} \\
r_{7}(n)=C r_{7}(m) \\
\text { where } C= \begin{cases}\left(40 \times 32^{\lambda}-9\right) / 31 & \text { if } m \equiv 1 \text { or } 2(\bmod 4), \\
\left(32^{\lambda+1}-1\right) / 31 & \text { if } m \equiv 3(\bmod 8), \\
\left(35 \times 32^{\lambda+1}+27\right) / 1147 & \text { if } m \equiv 7(\bmod 8),\end{cases} \\
r_{9}(n)=\frac{128^{\lambda+1}-1}{127} r_{9}(m) \quad \text { if } m \equiv 5(\bmod 8),
\end{gathered}
$$

and

$$
r_{11}(n)=\frac{512^{\lambda+1}-1}{511} r_{11}(m) \quad \text { if } m \equiv 7(\bmod 8) .
$$

Received 12th November, 2001.

Copyright Clearance Centre, Inc. Serial-fee code: 0004-9727/02 \$A2.00+0.00. 


\section{Proofs}

Let

$$
\phi(q)=\sum_{-\infty}^{\infty} q^{n^{2}}, \quad \psi(q)=\sum_{n \geqslant 0} q^{\left(n^{2}+n\right) / 2} .
$$

Following Cooper, we define $\phi_{s, t}$ for $s, t \geqslant 0$ by

$$
\phi_{s, t}=\sum_{n \geqslant 0} \phi_{s, t}(n) q^{n}=\phi(q)^{s} 2^{t} q^{t / 4} \psi\left(q^{2}\right)^{t}
$$

and we define $T$ by

$$
T \sum_{n \geqslant 0} u(n) q^{n}=\sum_{n \geqslant 0} u(4 n) q^{n}
$$

We shall require only

$$
\phi(q)=\phi\left(q^{4}\right)+2 q \psi\left(q^{8}\right), \phi(q)^{2}=\phi\left(q^{2}\right)^{2}+4 q \psi\left(q^{4}\right)^{2} \text { and } \psi(q)^{2}=\phi(q) \psi\left(q^{2}\right)
$$

Formulae RElating TO FIVE SQUARES

LEMma. For $\lambda \geqslant 0$

$$
T^{\lambda} \phi_{5,0}=\phi_{5,0}+\frac{5\left(8^{\lambda}-1\right)}{7} \phi_{1,4}
$$

Proof: We have

$$
\begin{aligned}
\phi_{5,0}= & \phi(q)^{5} \\
= & \left(\phi\left(q^{4}\right)+2 q \psi\left(q^{8}\right)\right)^{5} \\
= & \phi\left(q^{4}\right)^{5}+10 q \phi\left(q^{4}\right)^{4} \psi\left(q^{8}\right)+40 q^{2} \phi\left(q^{4}\right)^{3} \psi\left(q^{8}\right)^{2}+80 q^{3} \phi\left(q^{4}\right)^{2} \psi\left(q^{8}\right)^{3} \\
& +80 q^{4} \phi\left(q^{4}\right) \psi\left(q^{8}\right)^{4}+32 q^{5} \psi\left(q^{8}\right)^{5}
\end{aligned}
$$

from which it follows that

$$
\begin{aligned}
T \phi_{5,0} & =\phi(q)^{5}+80 q \phi(q) \psi\left(q^{2}\right)^{4} \\
& =\phi_{5,0}+5 \phi_{1,4}
\end{aligned}
$$

while

$$
\begin{aligned}
\phi_{1,4} & =16 q \phi(q) \psi\left(q^{2}\right)^{4} \\
& =16 q \phi(q) \phi\left(q^{2}\right)^{2} \psi\left(q^{4}\right)^{2}
\end{aligned}
$$




$$
\begin{aligned}
& =16 q\left(\phi\left(q^{4}\right)+2 q \psi\left(q^{8}\right)\right)\left(\phi\left(q^{4}\right)^{2}+4 q^{2} \psi\left(q^{8}\right)^{2}\right) \psi\left(q^{4}\right)^{2} \\
& =16 q\left(\phi\left(q^{4}\right)+2 q \psi\left(q^{8}\right)\right)\left(\phi\left(q^{4}\right)^{2}+4 q^{2} \psi\left(q^{8}\right)^{2}\right) \phi\left(q^{4}\right) \psi\left(q^{8}\right)
\end{aligned}
$$

from which it follows that

$$
\begin{aligned}
T \phi_{1,4} & =128 q \phi(q) \psi\left(q^{2}\right)^{4} \\
& =8 \phi_{1,4}
\end{aligned}
$$

Thus, with respect to the basis $\left\{\phi_{5,0}, \phi_{1,4}\right\}$ the matrix of $T$ is

$$
T=\left(\begin{array}{ll}
1 & 0 \\
5 & 8
\end{array}\right)
$$

and

$$
T^{\lambda}=\left(\begin{array}{cc}
\frac{1}{5\left(8^{\lambda}-1\right)} & 0 \\
7 & 8^{\lambda}
\end{array}\right) .
$$

The result follows.

\section{THEOREM .}

$$
\begin{aligned}
& \sum_{n \geqslant 0} r_{5}\left(4^{\lambda}(8 n+1)\right) q^{n}=\frac{10\left(8^{\lambda+1}-1\right)}{7}\left(\phi(q)^{4}+16 q \psi\left(q^{2}\right)^{4}\right) \psi(q), \\
& \sum_{n \geqslant 0} r_{5}\left(4^{\lambda}(8 n+5)\right) q^{n}=\frac{10 \times 8^{\lambda+2}+144}{7} \psi(q)^{5} \\
& \sum_{n \geqslant 0} r_{5}\left(4^{\lambda}(4 n+2)\right) q^{n}=\frac{20 \times 8^{\lambda+1}+120}{7} \phi(q)^{3} \psi\left(q^{2}\right)^{2}, \\
& \sum_{n \geqslant 0} r_{5}\left(4^{\lambda}(4 n+3)\right) q^{n}=\frac{5 \times 8^{\lambda+2}+240}{7} \phi(q)^{2} \psi\left(q^{2}\right)^{3} .
\end{aligned}
$$

Proof: We have

$$
\begin{aligned}
\sum_{n \geqslant 0} r_{5}\left(4^{\lambda} n\right) q^{n} & =T^{\lambda} \phi_{5,0}=\phi_{5,0}+\frac{5\left(8^{\lambda}-1\right)}{7} \phi_{1,4} \\
& =\phi(q)^{5}+\frac{80\left(8^{\lambda}-1\right)}{7} q \phi(q) \psi\left(q^{2}\right)^{4} \\
& =\phi(q)^{5}+\frac{80\left(8^{\lambda}-1\right)}{7} q \phi(q) \phi\left(q^{2}\right)^{2} \phi\left(q^{4}\right) \psi\left(q^{8}\right)
\end{aligned}
$$




$$
\begin{gathered}
=\left(\phi\left(q^{4}\right)+2 q \psi\left(q^{8}\right)\right)^{5}+\frac{80\left(8^{\lambda}-1\right)}{7} q\left(\phi\left(q^{4}\right)+2 q \psi\left(q^{8}\right)\right) \\
\left(\phi\left(q^{4}\right)^{2}+4 q^{2} \psi\left(q^{8}\right)^{2}\right) \phi\left(q^{4}\right) \psi\left(q^{8}\right) \\
=\phi\left(q^{4}\right)^{5}+\frac{10\left(8^{\lambda+1}-1\right)}{7} q \phi\left(q^{4}\right)^{4} \psi\left(q^{8}\right) \\
+\frac{20 \times 8^{\lambda+1}+120}{7} q^{2} \phi\left(q^{4}\right)^{3} \psi\left(q^{8}\right)^{2} \\
+\frac{5 \times 8^{\lambda+2}+240}{7} q^{3} \phi\left(q^{4}\right)^{2} \psi\left(q^{8}\right)^{3} \\
+\frac{80\left(8^{\lambda+1}-1\right)}{7} q^{4} \phi\left(q^{4}\right) \psi\left(q^{8}\right)^{4}+32 q^{5} \psi\left(q^{8}\right)^{5}
\end{gathered}
$$

It follows that

$$
\begin{aligned}
& \sum_{n \geqslant 0} r_{5}\left(4^{\lambda}(4 n+1)\right) q^{n}=\frac{10\left(8^{\lambda+1}-1\right)}{7} \phi(q)^{4} \psi\left(q^{2}\right)+32 q \psi\left(q^{2}\right)^{5} \\
& \sum_{n \geqslant 0} r_{5}\left(4^{\lambda}(4 n+2)\right) q^{n}=\frac{20 \times 8^{\lambda+1}+120}{7} \phi(q)^{3} \psi\left(q^{2}\right)^{2} \\
& \sum_{n \geqslant 0} r_{5}\left(4^{\lambda}(4 n+3)\right) q^{n}=\frac{5 \times 8^{\lambda+2}+240}{7} \phi(q)^{2} \psi\left(q^{2}\right)^{3}
\end{aligned}
$$

Further,

$$
\sum_{n \geqslant 0} r_{5}\left(4^{\lambda}(4 n+1)\right) q^{n}=\frac{10\left(8^{\lambda+1}-1\right)}{7}\left(\phi\left(q^{2}\right)^{2}+4 q \psi\left(q^{4}\right)^{2}\right)^{2} \psi\left(q^{2}\right)+32 q \psi\left(q^{2}\right)^{5}
$$

from which it follows that

$$
\begin{aligned}
& \sum_{n \geqslant 0} r_{5}\left(4^{\lambda}(8 n+1)\right) q^{n}=\frac{10\left(8^{\lambda+1}-1\right)}{7}\left(\phi(q)^{4}+16 q \psi\left(q^{2}\right)^{4}\right) \\
& \sum_{n \geqslant 0} r_{5}\left(4^{\lambda}(8 n+5)\right) q^{n}=\frac{10 \times 8^{\lambda+2}+144}{7} \psi(q)^{5}
\end{aligned}
$$

Corollary .

$$
\begin{aligned}
& r_{5}\left(4^{\lambda}(8 n+1)\right)=\frac{8^{\lambda+1}-1}{7} r_{5}(8 n+1), \\
& r_{5}\left(4^{\lambda}(8 n+5)\right)=\frac{5 \times 8^{\lambda+1}+9}{49} r_{5}(8 n+5),
\end{aligned}
$$




$$
\begin{aligned}
& r_{5}\left(4^{\lambda}(4 n+2)\right)=\frac{4 \times 8^{\lambda}+3}{7} r_{5}(4 n+2), \\
& r_{5}\left(4^{\lambda}(4 n+3)\right)=\frac{4 \times 8^{\lambda}+3}{7} r_{5}(4 n+3) .
\end{aligned}
$$

Proof: We have

$$
\sum_{n \geqslant 0} r_{5}\left(4^{\lambda}(8 n+1)\right) q^{n}=\frac{10\left(8^{\lambda+1}-1\right)}{7}\left(\phi(q)^{4}+16 q \psi\left(q^{2}\right)^{4}\right) .
$$

In particular

$$
\sum_{n \geqslant 0} r_{5}(8 n+1) q^{n}=10\left(\phi(q)^{4}+16 q \psi\left(q^{2}\right)^{4}\right)
$$

It follows that

The other results follow in the same way.

$$
r_{5}\left(4^{\lambda}(8 n+1)\right)=\frac{8^{\lambda+1}-1}{7} r_{5}(8 n+1) .
$$

\section{FormulaE RELATING TO SEVEN SQUARES}

LEMMA. With respect to the basis $\left\{\phi_{7,0}, \phi_{3,4}\right\}$,

$$
\begin{aligned}
T & =\left(\begin{array}{cc}
1 & 0 \\
35 & 32
\end{array}\right), \\
T^{\lambda} & =\left(\begin{array}{cc}
\frac{35\left(32^{\lambda}-1\right)}{31} & 32^{\lambda}
\end{array}\right) .
\end{aligned}
$$

THEOREM .

$$
\begin{aligned}
& \sum_{n \geqslant 0} r_{7}\left(4^{\lambda}(4 n+1)\right) q^{n}=\frac{560 \times 32^{\lambda}-126}{31} \phi(q)^{2} \psi\left(q^{2}\right)\left(\phi(q)^{4}+48 q \psi\left(q^{2}\right)^{4}\right) \\
& \sum_{n \geqslant 0} r_{7}\left(4^{\lambda}(4 n+2)\right) q^{n}=\frac{35 \times 32^{\lambda+1}-252}{31} \phi(q) \psi\left(q^{2}\right)^{2}\left(3 \phi(q)^{4}+16 q \psi\left(q^{2}\right)^{4}\right) \\
& \sum_{n \geqslant 0} r_{7}\left(4^{\lambda}(8 n+3)\right) q^{n}=\frac{280\left(32^{\lambda+1}-1\right)}{31}\left(\phi(q)^{4}+16 q \psi\left(q^{2}\right)^{4}\right) \psi(q)^{3} \\
& \sum_{n \geqslant 0} r_{7}\left(4^{\lambda}(8 n+7)\right) q^{n}=\frac{70 \times 32^{\lambda+2}+1728}{31} \psi(q)^{7}
\end{aligned}
$$

Corollary.

$$
r_{7}\left(4^{\lambda}(4 n+1)\right)=\frac{40 \times 32^{\lambda}-9}{31} r_{7}(4 n+1)
$$




$$
\begin{aligned}
& r_{7}\left(4^{\lambda}(4 n+2)\right)=\frac{40 \times 32^{\lambda}-9}{31} r_{7}(4 n+2), \\
& r_{7}\left(4^{\lambda}(8 n+3)\right)=\frac{32^{\lambda+1}-1}{31} r_{7}(8 n+3), \\
& r_{7}\left(4^{\lambda}(8 n+7)\right)=\frac{35 \times 32^{\lambda+1}+27}{1147} r_{7}(8 n+7) .
\end{aligned}
$$

We omit proofs of these results and those for nine and eleven squares since they are essentially the same as those for five squares.

\section{FormulaE RELATING TO NINE SQUARES}

LEMMA . With respect to the basis $\left\{\phi_{9,0}, \phi_{5,4}, \phi_{1,8}\right\}$,

$$
\begin{aligned}
T & =\left(\begin{array}{ccc}
1 & 0 & 0 \\
126 & 120 & 128 \\
9 & 8 & 0
\end{array}\right), \\
T^{\lambda} & =\left(\begin{array}{ccc}
1 & 0 \\
\frac{2160 \times 128^{\lambda}+254(-8)^{\lambda}-2414}{2159} & \frac{16 \times 128^{\lambda}+(-8)^{\lambda}}{17} & \frac{16\left(128^{\lambda}-(-8)^{\lambda}\right)}{17} \\
\frac{135 \times 128^{\lambda}-254(-8)^{\lambda}+119}{2159} & \frac{128^{\lambda}-(-8)^{\lambda}}{17} & \frac{128^{\lambda}+16(-8)^{\lambda}}{17}
\end{array}\right) .
\end{aligned}
$$

\section{THEOREM .}

$$
\begin{gathered}
\sum_{n \geqslant 0} r_{9}\left(4^{\lambda}(8 n+1)\right) q^{n} \\
=\frac{34560 \times 128^{\lambda}+4064(-8)^{\lambda}+238}{2159}\left(\phi(q)^{4}+16 q \psi\left(q^{2}\right)^{4}\right)^{2} \psi(q) \\
\quad+\frac{72990720 \times 128^{\lambda}-260096(-8)^{\lambda}+502656}{2159} q \psi(q)^{9}, \\
\sum_{n \geqslant 0} r_{9}\left(4^{\lambda}(8 n+5)\right) q^{n} \\
=\frac{4320\left(128^{\lambda+1}-1\right)}{127}\left(\phi(q)^{4}+16 q \psi\left(q^{2}\right)^{4}\right) \psi(q)^{5} \\
\sum_{n \geqslant 0} r_{9}\left(4^{\lambda}(4 n+2)\right) q^{n} \\
=\frac{380160 \times 128^{\lambda}-24384(-8)^{\lambda}-44880}{2159} \phi(q)^{7} \psi\left(q^{2}\right)^{2} \\
\quad+\frac{12718080 \times 128^{\lambda}+390144(-8)^{\lambda}-1501440}{2159} q \phi(q)^{3} \psi\left(q^{2}\right)^{6}
\end{gathered}
$$




$$
\begin{aligned}
= & \frac{1589760 \times 128^{\lambda}+48768(-8)^{\lambda}-187680}{2159} \phi(q)^{6} \psi\left(q^{2}\right)^{3} \\
& +\frac{12165120 \times 128^{\lambda}-780288(-8)^{\lambda}-1436160}{2159} q \phi(q)^{2} \psi\left(q^{2}\right)^{7} .
\end{aligned}
$$

COROLLARY .

$$
r_{9}\left(4^{\lambda}(8 n+5)\right)=\frac{128^{\lambda+1}-1}{127} r_{9}(8 n+5)
$$

Formulae RELATING TO ELEVEN SQUARES

LEMMA. With respect to the basis $\left\{\phi_{11,0}, \phi_{7,4}, \phi_{3,8}\right\}$,

$$
\begin{aligned}
T & =\left(\begin{array}{ccc}
1 & 0 & 0 \\
330 & 336 & 320 \\
165 & 176 & 192
\end{array}\right), \\
T^{\lambda} & =\left(\begin{array}{ccc}
\frac{9900 \times 512^{\lambda}+11242 \times 16^{\lambda}-21142}{15841} & \frac{20 \times 512^{\lambda}+11 \times 16^{\lambda}}{31} & \frac{20\left(512^{\lambda}-16^{\lambda}\right)}{31} \\
\frac{5445 \times 512^{\lambda}-11242 \times 16^{\lambda}+5797}{15841} & \frac{11\left(512^{\lambda}-16^{\lambda}\right)}{31} & \frac{11 \times 512^{\lambda}+20 \times 16^{\lambda}}{31}
\end{array}\right) .
\end{aligned}
$$

THEOREM .

$$
\begin{aligned}
\sum_{n \geqslant 0} r_{11}\left(4^{\lambda}(4 n+1)\right) q^{n} & \\
= & \frac{158400 \times 512^{\lambda}+179872 \times 16^{\lambda}+10230}{15841} \phi(q)^{10} \psi\left(q^{2}\right) \\
& \quad+\frac{219985920 \times 512^{\lambda}+14207424}{15841} q \phi(q)^{6} \psi\left(q^{2}\right)^{5} \\
& \quad+\frac{462274560 \times 512^{\lambda}-46047232 \times 16^{\lambda}+29855232}{15841} q^{2} \phi(q)^{2} \psi\left(q^{2}\right)^{9} \\
\sum_{n \geqslant 0} r_{11}\left(4^{\lambda}(4 n+2)\right) q^{n} & \\
= & \quad+\frac{4611520 \times 512^{\lambda}-359744 \times 16^{\lambda}+233244}{15841} \phi(q)^{9} \psi\left(q^{2}\right)^{2} \\
& +\frac{81100800 \times 512^{\lambda}+92094464 \times 16^{\lambda}+5237760}{15841} q^{2} \phi(q) \psi\left(q^{2}\right)^{10} \\
\sum_{n \geqslant 0} r_{11}\left(4^{\lambda}(8 n+3)\right) q^{n} &
\end{aligned}
$$




$$
\begin{gathered}
=\frac{22302720 \times 512^{\lambda}-1438976 \times 16^{\lambda}+46376}{15841}\left(\phi(q)^{4}+16 q \psi\left(q^{2}\right)^{4}\right)^{2} \psi(q)^{3} \\
+\frac{6617825280 \times 512^{\lambda}+92094464 \times 16^{\lambda}+13761024}{15841} q \psi(q)^{11} \\
\sum_{n \geqslant 0} r_{11}\left(4^{\lambda}(8 n+7)\right) q^{n}=\frac{63360\left(512^{\lambda+1}-1\right)}{511}\left(\phi(q)^{4}+16 q \psi\left(q^{2}\right)^{4}\right) \psi(q)^{7}
\end{gathered}
$$

Corollary .

$$
\begin{gathered}
r_{11}\left(4^{\lambda}(8 n+7)\right)=\frac{512^{\lambda+1}-1}{511} r_{11}(8 n+7) . \\
\text { REFERENCES }
\end{gathered}
$$

[1] S. Cooper, Sums of five, seven and nine squares, The Ramanujan Journal (to appear).

[2] S. Cooper, 'On the number of representations of certain integers as sums of eleven or thirteen squares', (submitted).

151, rue du chateau des Rentiers F-75013 Paris

France
School of Mathematics

UNSW

Sydney 2052

Australia

e-mail: m.hirschhorn@unsw.edu.au 